\title{
Effects of Resistance Training Frequency on Short-Term Neuromuscular Adaptations in Untrained Participants
}

\section{Rasmus K. Hansen, Rune R. Jørgensen, Peter S. Nielsen, Patrick Osuna-Florentz, Mathias Kristiansen}

Physical Activity and Human Performance Group-SMI, Department of Health Science and Technology, Aalborg University, Aalborg, Denmark

Email: Rasmuskopp@gmail.com

How to cite this paper: Hansen, R. K., Jørgensen, R. R., Nielsen, P. S., Osuna-Florentz, P., \& Kristiansen, M. (2017). Effects of Resistance Training Frequency on Short-Term Neuromuscular Adaptations in Untrained Participants. Advances in Physical Education, 7, 383-398. https://doi.org/10.4236/ape.2017.74032

Received: September 20, 2017

Accepted: October 31, 2017

Published: November 3, 2017

Copyright $\odot 2017$ by authors and Scientific Research Publishing Inc. This work is licensed under the Creative Commons Attribution International License (CC BY 4.0).

http://creativecommons.org/licenses/by/4.0/

c) (i) Open Access

\begin{abstract}
Objective: The purpose of this study was to evaluate whether performing resistance training four days per week was superior to two days per week, in promoting strength and neuromuscular adaptations of the leg extensor muscles. Methods: Nine untrained participants $(21.4 \pm 2.0$ years $)$ were pair matched according to gender and leg extensor maximal voluntary contraction (MVC) and randomly assigned to either a low frequency group (LFG) or a high frequency group (HFG). MVC, 10-repetition maximum (10 RM), and voluntary muscle activation (VA) were recorded pre and post two weeks of unilateral leg extension resistance training. The interpolated twitch technique was used to estimate VA and surface electromyography data was recorded during MVC. Results: VA and $10 \mathrm{RM}$ increased significantly for both groups. For LFG, VA increased from $84.5 \% \pm 5.0 \%$ to $92.5 \% \pm 3.4 \%(p=0.003)$ and for HFG, VA increased from $87.3 \% \pm 2.1 \%$ to $95.3 \% \pm 1.3 \%(p=0.006) .10$ $\mathrm{RM}$ increased from $43.2 \pm 8.0 \mathrm{~kg}$ to $48.2 \pm 6.2 \mathrm{~kg}(p=0.034)$ and from $45.3 \pm$ 5.9 to $49.0 \pm 7.4 \mathrm{~kg}(p=0.05)$ for LFG and HFG, respectively. No differences were observed between groups. Thus, two weeks of resistance training significantly increased the $10 \mathrm{RM}$ and VA in both groups, while no differences were observed between groups. Conclusions: These results indicate that performing resistance training two or four times per week are equally effective in promoting strength and neuromuscular adaptations, when weekly volume is equalized.
\end{abstract}

\section{Keywords}

Neural Adaptations, Strength Training Frequency, Resistance Training Frequency, Voluntary Activation 


\section{Introduction}

In resistance training programs, different variables can be manipulated in order to optimize training adaptations, and thus increase muscular strength (ACSM, 2009; Rhea et al., 2003). One variable that has gained interest in recent years is the frequency at which resistance training is performed (ACSM, 2009; Schoenfeld et al., 2016; Schoenfeld et al., 2015). Training frequency refers to the number of training sessions or the number of sessions a particular muscle or muscle group is trained during a given time period (Schoenfeld et al., 2016; Schoenfeld et al., 2015). The effects of different resistance training frequency on increases in strength have previously been investigated (Benton et al., 2011; Braith et al., 1989; Candow \& Burke, 2007; McLester et al., 2000; Schoenfeld et al., 2015).

For instance, Braith et al. (1989) investigated two vs. three days of single set resistance training and observed a significant increase in maximal voluntary contraction (MVC) in both groups. Yet, the group training two days per week only developed $80 \%$ of the strength increase compared to the group training three days per week, suggesting that a higher training frequency is more beneficial for increases in strength. However, it is important to note that weekly differences in training volume were not accounted for, resulting in both training volume and training frequency being higher in the group training three days per week. To account for this difference, Candow \& Burke (2007) implemented a volume-equalized study design and found that both two and three weekly resistance training sessions increased muscle strength and hypertrophy and that no differences between the groups were observed. Recently, Schoenfeld et al. (2015) investigated the effects of different resistance training frequencies by training either whole-body or split training with weekly volume being equalized for each muscle group. However, after the training period, no significant differences could be observed on muscular strength between the two groups. Accordingly, previous studies indicate that weekly volume rather than frequency may be more important when training for strength and hypertrophy (Benton et al., 2011; Braith et al., 1989; Candow \& Burke, 2007; McLester et al., 2000; Schoenfeld et al., 2015). Yet, the previous studies investigating the effects of resistance training frequency have focused on the training response to changes in strength and hypertrophy measured after several weeks (6 - 12 weeks) of resistance training. However, no information is available regarding the short-term effects ( $\leq$ three weeks) of training frequency on neuromuscular adaptations.

It is generally accepted that untrained individuals exhibit a rapid increases in maximal (Kamen \& Knight, 2004; Knight \& Kamen, 2001) and explosive (Aagaard et al., 2002) strength following a resistance training regimen. It has been argued that these early increments in strength can be attributed to neural adaptations (Braith et al., 1989; Folland \& Williams, 2007a; Gabriel et al., 2006). Surface electromyography (sEMG) has been used to assess muscle activation, and previous studies have shown increased sEMG amplitude following resistance training (Komi et al., 1978; Aagaard et al., 2002). Another method to investigate 
adaptations within the neuromuscular system is the interpolated twitch technique (ITT). This technique is used to investigate the degree of voluntary muscle activation (VA) (Folland \& Williams, 2007a; Gabriel et al., 2006; Knight \& Kamen, 2001; Merton, 1954; Place et al., 2010; Shield \& Zhou, 2004; Walker \& Häkkinen, 2014). Presently, the general consensus is that most muscles and muscle groups cannot be completely activated by voluntary effort in untrained individuals, which suggests that a force reserve is present (Folland \& Williams, 2007a). Any increase in the ability to voluntarily activate the muscle used in strength training could thus result in an increase in performance. However, in the literature, there are some inconsistencies regarding alterations in VA after a resistance training intervention, with some showing significant differences (Harridge et al., 1999) while others do not (Knight \& Kamen, 2001; Shima et al., 2002).

In summary, it seems that weekly volume rather than frequency per se is more important for long-term development of muscular strength and hypertrophy. However, as the evidence is sparse on the short-term effects of differences in frequency, it is not clear whether an optimal frequency for resistance training on neuromuscular adaptations exists. Accordingly, the purpose of the present study was to investigate the effects of resistance frequency with equal volume within the first two weeks of training. As neural adaptations are thought to have an important influence during the initial phase of a resistance training program, the aim of the present study was to: 1) examine whether differences in MVC, rate of force development (RFD) and 10 repetition maximum (RM) of the quadriceps femoris could be observed after two weeks of unilateral leg extensor resistance training; 2) investigate changes in VA and EMG amplitude; and 3) compare changes between the low and high training frequency group. Because of the more frequent stimulation of the neuromuscular system, we hypothesized that the high training frequency resistance training program would increase strength and measures of neural adaptations to a higher degree than the low training frequency group. Due to time constraints, the present study served as preliminary work and it will provide the basis for including a larger sample size in order to increase the statistical power of the study.

\section{Methods}

\subsection{Participants}

The participants enrolled in this study constituted a convenience sample and were enrolled by local advertisements. Thus, eight male and two female participants took part in the present investigation, which served as a preliminary study. None of the participants had been injured in the past six months or had any history of neuromuscular diseases that could influence the results. The participants had not performed organized resistance training (i.e. more than one training session per week) for the past six months. One subject was excluded due to technical issues at pre-test, and thus seven males and two females completed the 
study (see Table 1 for detailed information). The study was carried out in accordance with the ethical standards of the Helsinki Declaration and annual ethics approval from Aalborg University. Participants gave their written consent after having been explained the experimental methods and risks, both verbally and in writing. Throughout the duration of the study the participants were asked not to change their daily activity level or dietary habits. Moreover, the participants were instructed not to consume alcohol or caffeine in the 24 hours leading up to each test.

\subsection{Familiarization and Groups}

The participants attended one familiarization session, with the purpose of habituating them to the testing procedures and to ensure that they could reliably perform all types of muscle contractions utilized in the test. Each participant performed several isometric and isokinetic contractions and was also familiarized to the ITT procedure. Participants were randomized into two groups, a high frequency group (HFG) that trained four times per week and a low frequency group (LFG) that trained two times per week. The participants were matched in pairs according to their gender and MVC obtained at the pre-test and then randomly allocated to either LFG $(n=5)$ or HFG $(n=4)$. The training period for both groups was two weeks.

\subsection{Training Protocol}

LFG trained unilateral leg extension for the right leg two times per week, with each session including four sets of $10-12$ repetitions of $\sim 60 \% 1$ RM. Additionally, four sets of five seconds MVC with a knee angle of $65^{\circ}$ was performed. The training sessions were separated by 48 - 72 hours.

HFG trained unilateral leg extension for the right leg four times per week, with each session including two sets of $10-12$ repetitions of $\sim 60 \% 1$ RM. Additionally, two sets of five seconds MVC with a knee angle of $65^{\circ}$ was performed. The training sessions were separated by 24 - 48 hours.

At each training session, the participants in both groups trained to concentric failure. If more than the targeted repetitions could be performed, more weight was added. Movement velocity was controlled by a metronome set at $0.5 \mathrm{~Hz}$, yielding a two second concentric and a two second eccentric phase of the dynamic

Table 1. The table shows the descriptive data for the participants.

\begin{tabular}{cccc}
\hline & LFG $(\mathrm{n}=5)$ & HFG $(\mathrm{n}=4)$ & $p$-value \\
\hline Age (yrs) & $20.8 \pm 1.8$ & $22.3 \pm 2.2$ & 0.312 \\
Height (m) & $1.87 \pm 0.08$ & $1.74 \pm 0.10$ & 0.072 \\
Body mass (kg) & $84.7 \pm 17.5$ & $81.2 \pm 18.4$ & 0.778 \\
Body fat (\%) & $19.6 \pm 10.0$ & $20.2 \pm 6.9$ & 0.617
\end{tabular}


knee extension. A two-minute rest interval was applied between all sets. Each training session was initiated with a brief warm-up session consisting of five minutes on a bicycle ergometer using a self-selected intensity and cadence followed by a warm-up set consisting of 10 repetitions at $60 \%$ of the load of the last working set from the previous training session. The training protocol in the two groups was designed based on existing recommendations for development in strength in untrained individuals (ACSM, 2009). On the first and last training day, a 10 RM test was performed to assess dynamic strength. For each training session the amount of repetitions and weight lifted in total was noted. The training was performed on a leg extension machine (Life Fitness SL25 Leg Extension, Illinois, USA). When performing the leg extension exercise the machine was set up, so that the participants' back was firmly placed on the backrest. Moreover, the fulcrum of the knee was aligned with the fulcrum of the lever arm of the leg extension machine and the leg cushion was located just above the lateral malleolus of the right leg. The settings were saved for each participant, and re-used each training session.

\subsection{Test Protocol}

A Kinetic Communicator 125E dynamometer (Kin-Com) (Chattecx Corporation, Chattanooga, TN, USA, Model 500-11 with software version 3.21) was used to measure isometric and isokinetic strength during unilateral leg extension of the right leg. All data was sampled using a sampling frequency of $100 \mathrm{~Hz}$. The Kin-Com was set up individually to each participant. This included the length of the lever arm according to the length of the lower leg and gravity compensation for the weight of the leg was performed at the beginning of all test sessions. The distal end of the load cell was located just above the lateral malleolus.

Participants were seated with a $90^{\circ}$ hip flexion on the associated chair and fixated at the chest, contralateral thigh, and hip to increase the validity and reliability (Brown \& Weir, 2001). The settings for each participant was saved and used again for the post-test. At each test visual feedback of the force output and verbal encouragement were provided.

\subsection{MVC and RFD Measurement}

The Kin-Com was set to measure isometric force production for the right leg at a knee angle of $65^{\circ}$. The MVC contractions were also used to calculate the RFD. Prior to the measurements a standardized warm-up was performed. The warm-up consisted of a progressive force increase with two contractions for five seconds at 50,70, and one at $90 \%$ of self-perceived MVC. The warm-up was performed to ensure the participant were ready to perform maximally (Aagaard et al., 2002). At the MVC trials each participant was instructed to "contract as hard and fast as possible" and then performed a maximal contraction for 5 seconds. Three trials were acquired with a resting interval of two minutes between each trial. The highest value was used in the data analyses. 


\subsection{Isokinetic Testing}

The Kin-Com was set to measure isokinetic force production at the right leg from $0^{\circ}$ to $90^{\circ}$ and $90^{\circ}$ to $0^{\circ}$ at $45^{\circ} / \mathrm{sec}$ for the CON and ECC part, respectively. The $45^{\circ} / \mathrm{sec}$ was chosen to mimic the training sessions (i.e. 2:2 sec, CON and ECC) as training adaptations have been shown to be velocity specific (Rutherford \& Jones, 1986).

Prior to all measurements a standardized warm-up was performed. The warm-up consisted of two isokinetic submaximal CON and ECC contractions at $50 \%$ and two CON and ECC contractions at $100 \%$. At the test the participant was instructed to "contract as hard as possible" and then performed a CON followed by an ECC contraction, separated by a two-minute rest interval between each set.

\subsection{Interpolated Twitch Technique}

The transcutaneous electrical stimulus consisted of a high voltage $(400 \mathrm{~V})$ constant current stimulator (DS7AH; Digitimer, Hertfordshire, United Kingdom) delivered to the femoral nerve. The location of the hot spot for stimulating the femoral nerve was determined with a probe with an intensity of $20 \mathrm{~mA}, 400 \mathrm{~V}$ and a duration of $500 \mu \mathrm{s}$. The spot that yielded the highest twitch force response was selected and a self-adhesive circular cathode (diameter $3.2 \mathrm{~cm}$ PALS, Axelgaard, CA, United States) was placed at the hot spot. Furthermore, an anode $(5 \times$

$9 \mathrm{~cm}$ PALS, Axelgaard, CA, United States) was placed at the gluteal sulcus (Place et al., 2010).

During the test procedure, the stimuli was delivered as a single-pulse stimulus (Shield \& Zhou, 2004).

It has been suggested that a single-pulse stimulus is just as effective as doublets or trains of stimuli, in evoking complete activation while simultaneously decreasing the unpleasantness felt by the participants (Shield \& Zhou, 2004).

The maximal stimulation responses of each participant were determined with incremental amperage $(1-100 \mathrm{~mA})$. The amperage that produced the highest peak twitch force was multiplied by 1.5 to ensure a supramaximal stimuli.

During the tests, a supramaximal superimposed stimulation was delivered during the peak plateau of the MVC ( 1 - 5 seconds) determined by visual detection. Another stimulus was delivered 10 seconds after contraction at rest, to elicit a control twitch. The same investigator administered all the trials to increase the test-retest reliability. To ensure the participants performed maximally, a rejection criteria was administered, as recommended by Shield and Zhou (2004), and if these criteria were not fulfilled the contraction was not accepted and thus repeated. The rejection criteria were as follows: 1) If no force plateau was observed or 2) if the force production was considered submaximal by the subject.

\subsection{Electromyography}

During the isometric and isokinetic tests the muscle activity of Vastus Lateralis 
(VL), Vastus Medialis (VM) and Rectus Femoris (RF) were evaluated by recording sEMG data. Before electrode placement, the skin area was shaved, abraded and cleaned with alcohol. When the alcohol had vaporized the electrodes (Ambu Neuroline 720 01-K/12, Ag/AgCl, inter electrode distance $20 \mathrm{~mm}$, Ambu A/S, Ballerup, Denmark) were mounted along the muscle fiber direction in a bipolar configuration. The surface electrodes were placed in accordance with the recommendations by SENIAM. A reference electrode was placed on the lateral epicondyle of the right knee. sEMG data were collected at a sampling rate of $2000 \mathrm{~Hz}$, band-pass filtered $(10-500 \mathrm{~Hz}$ ), and amplified by $1000 \mathrm{~Hz}$ using a custom made LabVIEW@-based computer program (Kick@ v2.030e, University of Aalborg, Aalborg, Denmark).

\subsection{Data Analyses}

1) $M V C$ : The trial with the highest maximal force value obtained from the three attempts was chosen. For calculation of maximal voluntary force $(\mathrm{N})$ the highest average over 1 second was reported as MVC (De Luca, 1997; Knight \& Kamen, 2001; Maffiuletti \& Martin, 2000).

2) RFD: The RFD (N/s) was calculated as the slope of the force time curve ( $\Delta$ force/ $\Delta$ time) and was obtained from the same three contractions as the MVC trials. RFD was calculated at time bands of 0 - 30, 0 - 50, 0 - 90, 0 - 100, 0 - 150, 0 - 200 and 0 - $250 \mathrm{~ms}$ (Haff et al., 2015) where RFD in each time band were derived as the average slope of the force-time curve for that band. For $\mathrm{RFD}_{\text {peak }}$, the average slope over the highest successive $20 \mathrm{~ms}$ period was reported (Haff et al., 2015).

3) Isokinetic CON and ECC: During the isokinetic trials, average CON and ECC force $(\mathrm{N})$ over the entire $90^{\circ}$ range of motion were calculated. The average of the three trials was reported as ISOK CON and ISOK ECC.

4) ITT: After identification of the superimposed and control twitch for each of the three contractions, a triggered average was performed to reduce background noise (Dowling et al., 1994). Accordingly, the VA was calculated using the following equation (Shield \& Zhou, 2004):

$$
\begin{aligned}
& \text { Voluntary activation }(\%) \\
& =(1-(\text { superimposed twitch/control twitch })) \times 100
\end{aligned}
$$

5) $E M G$ : During MVC, ISOK CON, and ISOK ECC trials, root mean square (RMS) was calculated as an index of EMG amplitude for each muscle (RF, VL and VM) (Andersen, Christensen, Samani, \& Madeleine, 2014; De Luca, 1997). The RMS value of the highest consecutive epoch of $250 \mathrm{~ms}$ was calculated (Andersen et al., 2014; Vangsgaard, Taylor, Hansen, \& Madeleine, 2014) with a $50 \%$ overlap between epochs (Andersen et al., 2014). For all the muscles, the highest RMS value over the three trials was reported as $\mathrm{RMS}_{\max }$. All data was processed and analyzed in MATLAB (Mathworks R2014b, Kista, Sweden) and then used for statistical analyses. 


\subsection{Statistical Analysis}

A Shapiro Wilks test was used to test all data for normality. Descriptive data of HFG and LFG was compared using an independent sample T-test. A two way repeated measures ANOVA with time and group as factors was used to compare pre- and post-test within and between the two groups. If a significant difference was found for the time or group factor, a dependent- or independent samples T-test was used as a post hoc. All calculations were done in SPSS (SPSS ${ }^{\varpi}$ Statistics v. 23.0.0.0 64-bit edition, IBM $^{\bowtie}$ Armonk, New York, USA). The level of significance was set to $p \leq 0.05$ and $p$-values are reported for all measures. For $\mathrm{RFD}_{\text {bands }}$ and EMG the $p$-values are given as a range.

\section{Results}

Nine participants completed all testing procedures. No significant differences were found between groups for age, height, body mass and body composition $(p>0.05)$ (see Table 1). Furthermore, the total number of lifts and total volume lifted during all training sessions were not significantly different between groups $(p>0.05)$ (see Table 2).

\subsubsection{RM Strength}

The results for $10 \mathrm{RM}$ strength are shown in Figure 1. A significant difference within groups was observed $(p=0.004)$.

Table 2. 10 RM pre- and post-test, total number of lift and total volume lifted during all training sessions are shown for LFG and HFG and their $p$-value.

\begin{tabular}{cccc}
\hline & LFG $(\mathrm{n}=5)$ & HFG $(\mathrm{n}=4)$ & $p$-value \\
\hline $\begin{array}{c}\text { 10 RM pre-test to post-test } \\
(\mathrm{kg})\end{array}$ & $43.1 \pm 8.0-48.2 \pm 7.4$ & $45.3 \pm 5.9-49.0 \pm 6.2$ & $\begin{array}{c}\text { LFG: } 0.033, \\
\text { HFG: } 0.037\end{array}$ \\
Total number of lifts & $166.4 \pm 8.6$ & $181.5 \pm 19.9$ & LFG and \\
HFG: $>0.05$
\end{tabular}

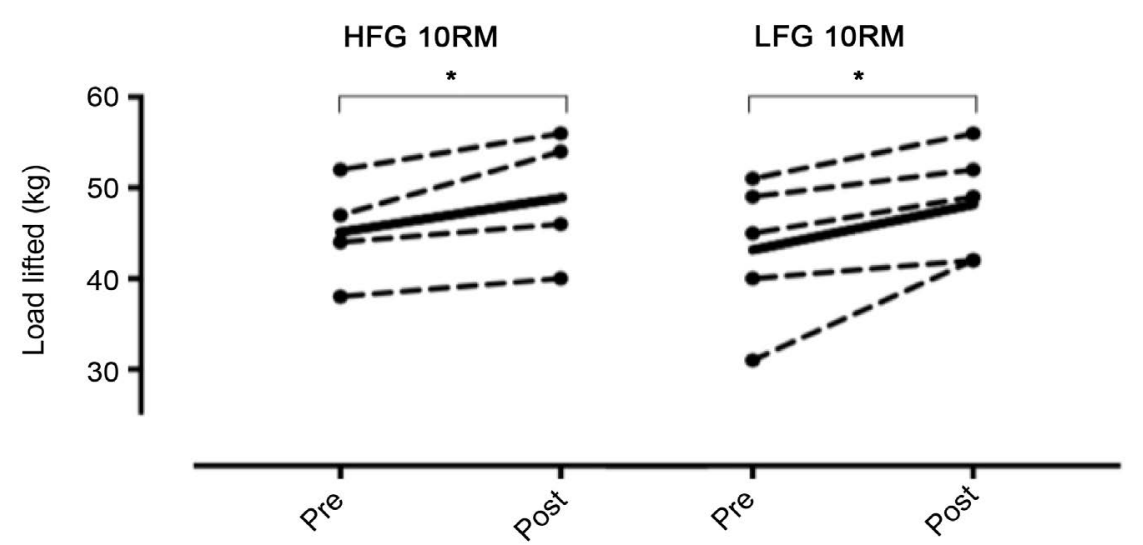

Figure 1. 10 RM strength for HFG and LFG. ${ }^{*} p<0.05$. 
HFG significantly increased 10RM strength from pre- to post-test by $45.3 \pm$ $5.9 \mathrm{~kg}$ to $49.0 \pm 7.4 \mathrm{~kg}(p=0.050)$.

Furthermore, LFG significantly increased $10 \mathrm{RM}$ strength from pre- to post-test by $43.2 \pm 8.0 \mathrm{~kg}$ to $48.2 \pm 6.2 \mathrm{~kg}(p=0.034)$. No significant difference was found between groups $(p=0.565)$. See Figure 1 and Table 2 .

\subsection{Voluntary Activation}

The results for VA are shown in Figure 2. A significant difference was found within $(p \leq 0.001)$, but not between groups $(p=0.994)$. HFG significantly increased their VA from pre to post-test by $87.3 \% \pm 2.1 \%$ to $95.3 \% \pm 1.3 \%$ ( $p=$ 0.006)

LFG significantly increased their VA from pre to post-test by $84.5 \% \pm 5.0 \%$ to $92.5 \% \pm 3.4 \%(p=0.003)$. One participant was excluded from the VA measurements due to technical problems at the post-test.

\subsection{Maximum Voluntary Contraction}

No significant difference was found from pre- to post-test for either HFG (718.9 \pm $108.6 \mathrm{~N}$ to $770.0 \pm 147.9 \mathrm{~N})$ or LFG $(702.8 \pm 89.2 \mathrm{~N}$ to $716.9 \pm 153.4 \mathrm{~N})(p=$ $0.340)$. Moreover, no difference was found between groups $(p=0.579)$.

\subsection{Isokinetic Strength}

No significant difference was found from pre- to post-test in ISOK CON (HFG: $325.3 \pm 92.0 \mathrm{~N}$ to $381.6 \pm 94.9 \mathrm{~N}$ and LFG: $352.2 \pm 32.7$ to $328.1 \pm 59.9 \mathrm{~N})(p=$ $0.555)$ or ISOK ECC (HFG: $418.1 \pm 104.0$ to $500.2 \pm 126.1 \mathrm{~N}$, LFG: $451.8 \pm 36.4$ to $450.7 \pm 49.4 \mathrm{~N})(p=0.202)$ or between groups for ISOK CON $(p=0.164)$ and ISOK ECC $(p=0.192)$.

\subsection{Rate of Force Development}

For $\mathrm{HFG}, \mathrm{RFD}_{\text {peak }}$ was $13,975.0 \pm 1916.8 \mathrm{~N} / \mathrm{s}$ and $13,925.0 \pm 3124.5 \mathrm{~N} / \mathrm{s}$ in the

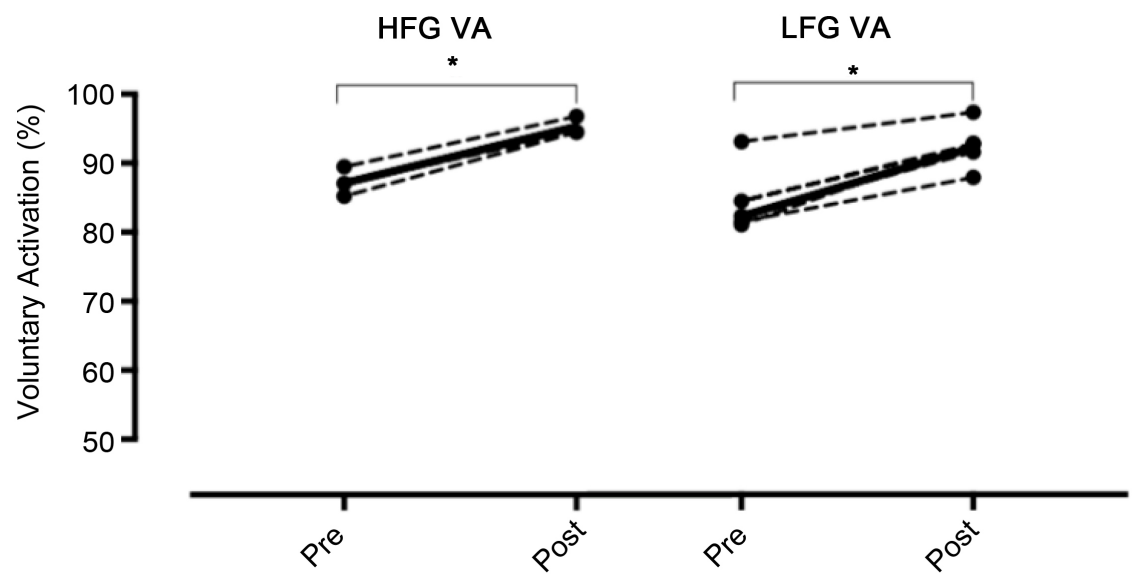

Figure 2. Voluntary activation (VA) depicted for HFG and LFG for the pre and post-test. ${ }^{*} p<0.05$. 
pre- and post-test, respectively.

For LFG, RFD peak was 10,700.0 $\pm 4513.0 \mathrm{~N} / \mathrm{s}$ and $9760.0 \pm 2802.3 \mathrm{~N} / \mathrm{s}$ in the pre- and post-test, respectively. No significant difference was found from pre- to post-test within $(p=0.613)$ or between groups in $\operatorname{RFD}_{\text {peak }}(p=0.649)$ (see Figure 3$)$.

\subsection{Electromyography}

A significant increase was found within groups for ISOK ECC in LFG in VL from $253.1 \pm 128.1 \mu \mathrm{V}$ to $343 \pm 140 \mu \mathrm{V}(p=0.004)$ and for MVC in VL from $317.3 \pm 185.4 \mu \mathrm{V}$ to $449 \pm 256.7 \mu \mathrm{V}(p=0.027)$ but no difference was found for RF and VM ( $p=0.198-0.978)$ from pre- to post-test in LFG. No other significant differences were found.

\section{Discussion}

The aim of the present study was to examine whether differences in leg extensor strength could be observed after two weeks of unilateral leg extensor resistance training performed on two or four days per week with equalized training volume. The main finding of the present study was that two weeks of resistance training significantly increased VA and $10 \mathrm{RM}$ in both groups and that no differences were observed between groups in any of the investigated parameters. These results could indicate that training two or four days per week promotes similar adaptations when training volume is equalized, at least during the first two weeks of resistance training.

RFD

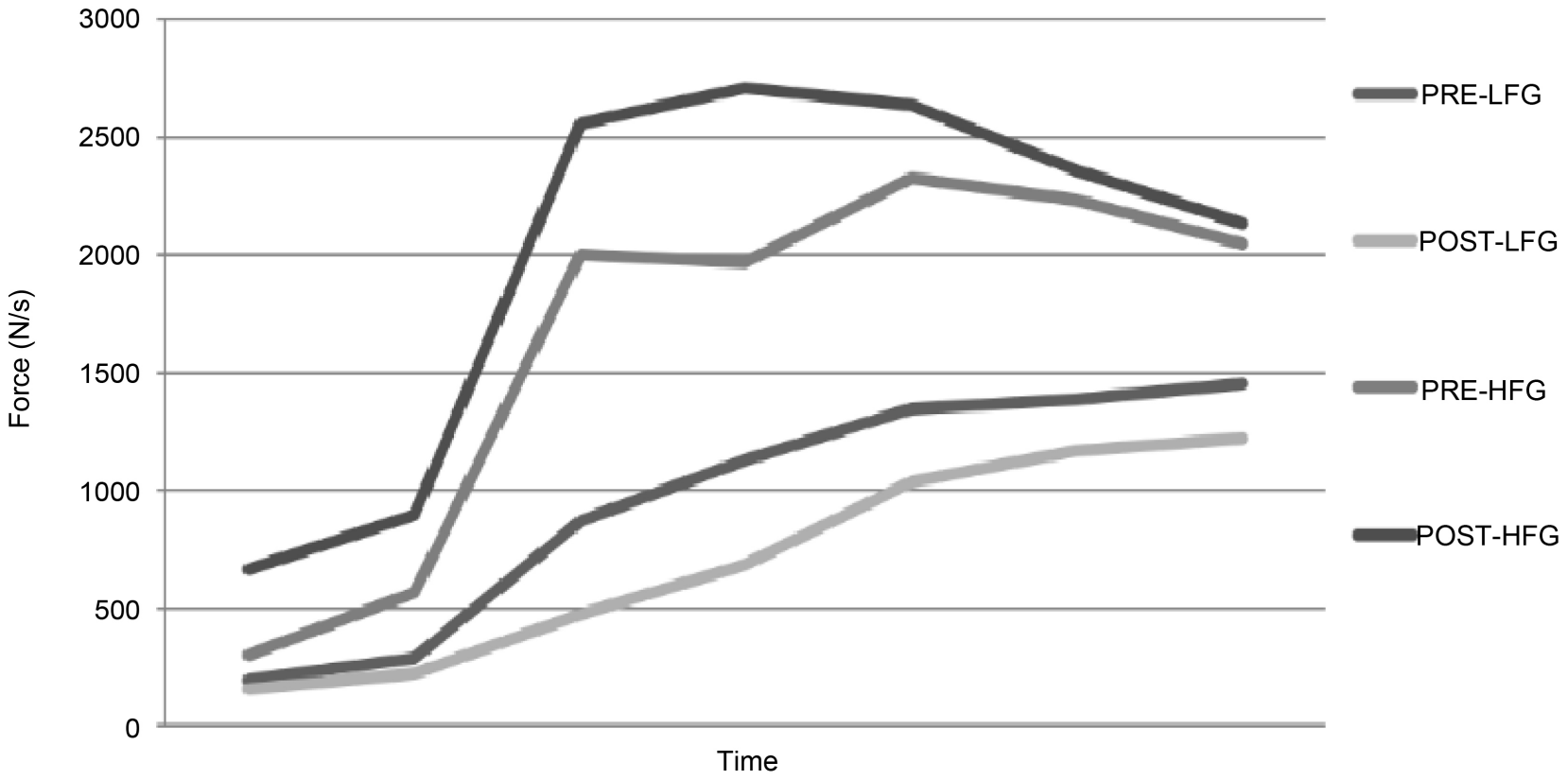

Figure 3. Rate of force development (RFD) for HFG and LFG during pre and post-test. 


\subsection{Dynamic and Isometric Strength}

1) 10 RM Strength: The main finding of the dynamic strength assessment was that a significant increase in 10 RM strength was observed in LFG and HFG, which is in line with previous findings (Komi et al., 1978).

2) $M V C$ : No significant increases in MVC were observed for LFG or HFG. This finding is in contrast to earlier studies investigating neuromuscular adaptations in untrained individuals (Kamen \& Knight, 2004; Knight \& Kamen, 2001). After implementing both dynamic and isometric leg extension, Kamen \& Knight (Kamen \& Knight, 2004) and Knight \& Kamen (Knight \& Kamen, 2001) found significant increases in MVC force after six weeks. As the resistance training program used in both of the before mentioned studies resembles the one used in the present study, the design of the program is unlikely to have caused this discrepancy. However, another explanation for the difference in MVC might be differences in training volume or duration of the intervention. While the weekly training volume was almost equal between the studies (Kamen \& Knight, 2004; Knight \& Kamen, 2001) and the present study, the duration of the present study markedly differed. A significant increase was found after six weeks, but after two weeks no differences were detected (Kamen \& Knight, 2004; Knight \& Kamen, 2001). The fact that two weeks of resistance training was not enough to elicit a significant increase is similar to the results of the present study. It is possible that the training intervention should have incorporated a larger volume of MVC trials to elicit positive adaptations within this specific test. As training adaptations are specific to the training being performed (Rutherford \& Jones, 1986), this may serve as an explanation of the observed increase in 10RM load, but not in MVC. Rutherford and Jones (1986) found that, relative to isometric strength, the increase in dynamic strength was larger after a dynamic resistance training program and similar that after an isometric resistance training program, the increase in isometric strength was greater than the increase in dynamic strength. Specificity of the training coincides well with the observation that in the present study, the pooled $10 \mathrm{RM}$ strength tended to increase more $(10.9 \% \pm 9.8 \%)$ than the pooled MVC $(4.1 \% \pm 12.9 \%)$, although this was not significant $(p=0.148)$.

3) RFD: No differences were observed between LFG and HFG in either RFD peak or any of the $\mathrm{RFD}_{\text {bands }}$. Moreover, no difference was observed from pre- to post-test in either of the groups. Previous studies have found an increase in RFD after resistance training (Aagaard et al., 2002). Some of the mechanisms that are thought to be responsible for increases in maximal force have also been suggested to be responsible for increases in explosive force development (Maffiuletti et al., 2016). As this study did not find a significant increase in MVC, this might explain why RFD did not change either. Moreover, as both heavy and ballistic resistance training have been shown to increase RFD (Maffiuletti et al., 2016), it is possible that the $\sim 60 \%$ of $1 \mathrm{RM}$ with moderate (2:2 second) contraction velocity was to light and/or to slow to significantly alter RFD, as it previously have been found that adaptations to resistance training are velocity-specific (Farth- 
ing \& Chilibeck, 2003).

\subsection{Electromyography}

$E M G$ : In the present study we found a significant increase for the LFG in $\mathrm{RMS}_{\max }$ of the VL during the ISOK ECC and MVC trials. This result is in contrast to Rabita et al. (2000) who found a significant increase in RMS amplitude of the RF during MVC, but with no change in VM or VL. However, one explanation for the different response could be that the participants only trained isometric in the study by Rabita et al. (2000) whereas the present resistance training program also included dynamic training. This may suggest that the different parts of the quadriceps femoris complex adapts differentially to different resistance training regimes, supporting the observation of velocity-specific adaptations (Farthing \& Chilibeck, 2003). Yet, an explanation to why LFG increased in ISOK ECC

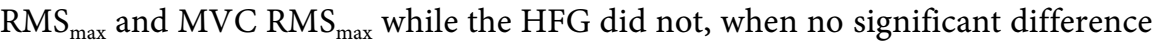
was observed in the development in ISOK ECC and MVC force, could be that the HFG improved in other neural aspects, which could not be detected in the present design.

\subsection{Voluntary Activation}

$V A$ : Both LFG and HFG significantly increased VA with no difference between the groups. Because the training volume was the same in the LFG and HFG and no difference in VA between the groups was observed, this could indicate that the change in VA is more sensitive to the overall volume of training rather than how the training is dispersed. The finding of an increase in VA after a short-term resistance training program is in agreement with some (Knight \& Kamen, 2001; Shima et al., 2002) but in contrast to others (Harridge et al., 1999). One of the possible mechanisms for increases in VA and EMG has been suggested to be an increase in agonist activity (Walker \& Häkkinen, 2014). Increased agonist activity has been attributed to increased motor unit firing rate (Christie \& Kamen, 2010; Kamen \& Knight, 2004; Patten et al., 2001; Rich \& Cafarelli, 2000; Van Cutsem et al., 1998), doublet firing, synchronization (Van Cutsem et al., 1998) and recruitment (De Luca, 1997; Walker \& Häkkinen, 2014) and it is likely that some of these mechanisms can explain the changes in $\mathrm{RMS}_{\max }$ and VA, as well as 10RM strength. The apparent discrepancy between increases in VA, and no alterations in MVC performance presented in this study is in contrast to previous studies (Knight \& Kamen, 2001; Scaglioni et al., 2002; Shima et al., 2002), where increases in VA and MVC are shown to occur concurrently. It is generally agreed that the VA is indicative of an individual's ability to voluntarily recruit motor units. As most of the training performed in the current study was done as dynamic leg extension training, and only a small part of the training was performed as isometric contractions, it may be possible that the ability to recruit motor units during leg extension movement increased as evidenced by the significant strength increase in $10 \mathrm{RM}$. However, due to the very short train- 
ing period and very low isometric training volume, this may not have had a functional impact on the MVC performance.

\subsection{Methodological Considerations}

In the present study, the EMG amplitude was reported in absolute values. It can be seen as a limitation that no normalization procedure was made in the present study, as this technique has been reported to enhance comparison within and between individuals (De Luca, 1997). However, it has previously been shown that absolute RMS values tend to be more reliable than the normalized values (Andersen et al., 2014).

The inclusion of women is in agreement with other studies investigating the effects of different resistance training frequencies (Braith et al., 1989; Candow \& Burke, 2007), although it has previously been speculated that women may adapt differently than men to training (Folland \& Williams, 2007b). However, the study by Candow \& Burke (2007) found no gender and time interaction, suggesting no differences in change of strength over the six weeks of the resistance training program between genders.

It can be seen as a limitation that no measures of hypertrophy were performed. Even though morphological adaptations are occurring readily after the first bout of exercise (Folland \& Williams, 2007b), the extent of these are probably not a major contributor during the first few weeks of training, as it has been found that the first weeks of strength progression is primarily due to neural and not morphological adaptations (Griffin \& Cafarelli, 2005).

In conclusion, the present study demonstrated a significant increase in VA in both groups, suggesting an increased neural drive. Moreover, both groups increased in $10 \mathrm{RM}$ strength, but with no significant difference between the groups in any of the strength assessments. These results suggest that both two and four days per week are effective in promoting the neural adaptations attributed to the early phase of a resistance training program. Thus, our hypothesis that four days per week would increase strength and neural adaptations more than two days per week was not confirmed, however due to the preliminary nature of the present study, and thus the small sample size, caution should be taken before any definitive conclusions are made.

\section{Conflict of Interest}

The authors have no conflict of interest.

\section{References}

Aagaard, P., Simonsen, E. B., Andersen, J. L., Magnusson, P., \& Dyhre-Poulsen, P. (2002). Increased Rate of Force Development and Neural Drive of Human Skeletal Muscle Following Resistance Training. Journal of Applied Physiology, 93, 1318-1326. https://doi.org/10.1152/japplphysiol.00283.2002

ACSM (2009). Progression Models in Resistance Training for Healthy Adults. Medicine 
and Science in Sports and Exercise, 22, 179-182.

Andersen, K. S., Christensen, B. H., Samani, A., \& Madeleine, P. (2014). Between-Day Reliability of a Hand-Held Dynamometer and Surface Electromyography Recordings during Isometric Submaximal Contractions in Different Shoulder Positions. Journal of Electromyography and Kinesiology, 24, 579-587.

https://doi.org/10.1016/j.jelekin.2014.05.007

Benton, M. J., Kasper, M. J., Raab, S. A., Waggener, G. T., \& Swan, P. D. (2011). Short-Term Effects of Resistance Training Frequency on Body Composition and Strength in Middle-Aged Women. Journal of Strength \& Conditioning Research, 25, 3142-3149. https://doi.org/10.1519/JSC.0b013e31820f505f

Braith, R. W., Graves, J. E., Pollock, M. L., Leggett, S. L., Carpenter, D. M., \& Colvin, A. B. (1989). Comparison of 2 vs. 3 Days/Week of Variable Resistance Training during 10and 18-Week Programs. International Journal of Sports Medicine, 10, 450-454.

https://doi.org/10.1055/s-2007-1024942

Brown, L. E., \& Weir, J.P. (2001). ASEP Procedures Recommendation I: Accurate Assessment of Muscular Strength and Power. Journal of Exercise Physiology, 4, 1-21.

Candow, D. G., \& Burke, D. G. (2007). Effect of Short-Term Equal-Volume Resistance Training with Different Workout Frequency on Muscle Mass and Strength in Untrained Men and Women. Journal of Strength \& Conditioning Research, 21, 204-207. https://doi.org/10.1519/00124278-200702000-00037

Christie, A., \& Kamen, G. (2010). Short-Term Training Adaptations in Maximal Motor Unit Firing Rates and after Hyperpolarization Duration. Muscle and Nerve, 41, 651-660.

De Luca, C. J. (1997). The Use of Surface Electromyography in Biomechanics. Journal of Applied Biomechanics, 13, 1-38. https://doi.org/10.1123/jab.13.2.135

Dowling, J. J., Konert, E., \& Andrews, D. M. (1994). Are Humans Able to Voluntarily Elicit Maximum Muscle Force? Neuroscience Letters, 179, 25-28.

Farthing, J. P., \& Chilibeck, P. D. (2003). The Effect of Eccentric Training at Different Velocities on Cross-Education. European Journal of Applied Physiology, 89, 570-577. https://doi.org/10.1007/s00421-003-0841-3

Folland, J. P., \& Williams, A. G. (2007a). Methodological Issues with the Interpolated Twitch Technique. Journal of Electromyography and Kinesiology, 17, 317-327.

Folland, J. P., \& Williams, A. G. (2007b). The Adaptations to Strength Training. Sports Medicine, 37, 145-168. https://doi.org/10.2165/00007256-200737020-00004

Gabriel, D. A., Kamen, G., \& Frost, G. (2006). Neural Adaptations to Resistive Exercise: Mechanisms and Recommendations for Training Practices. Sports Medicine, 36, 133-149. https://doi.org/10.2165/00007256-200636020-00004

Griffin, L., \& Cafarelli, E. (2005). Resistance Training: Cortical, Spinal and Motor Unit Adaptations. Canadian Journal of Applied Physiology, 30, 328-340. https://doi.org/10.1139/h05-125

Haff, G. G., Ruben, R. P., Lider, J., Twine, C., \& Cormie, P. A. (2015). Comparison of Methods for Determining the Rate of Force Development during Isometric Midthigh Clean Pulls. The Journal of Strength \& Conditioning Research, 29, 386-395. https://doi.org/10.1519/JSC.0000000000000705

Harridge, S. D. R., Kryger, A., \& Stensgaard, A. (1999). Knee Extensor Strength, Activation, and Size in Very Elderly People Following Strength Training. Muscle and Nerve, 22, 831-839.

https://doi.org/10.1002/(SICI)1097-4598(199907)22:7<831::AID-MUS4>3.0.CO;2-3 
Kamen, G., \& Knight, C. A. (2004). Training-Related Adaptations in Motor Unit Discharge Rate in Young and Older Adults. The Journal of Gerontology, Series A: Biolog ical Sciences and Medical Sciences, 59, 1334-1338. https://doi.org/10.1093/gerona/59.12.1334

Knight, C. A., \& Kamen, G. (2001). Adaptations in Muscular Activation of the Knee Extensor Muscles with Strength Training in Young and Older Adults. Journal of Electromyography and Kinesiology, 11, 405-412.

Komi, P. V., Viiasalo, J. T., Rauramaa, R., \& Vihko, V. (1978). Effect of Isometric Strength Training on Mechanical, Electrical, and Metabolic Aspects of Muscle Function. European Journal of Applied Physiology, 40, 45-55. https://doi.org/10.1007/BF00420988

Maffiuletti, N. A., \& Martin, A. (2000). Progressive versus Rapid Rate of Contraction during 7 wk of Isometric Resistance Training. Medicine \& Science in Sports \& Exercise, 33, 1220-1227.

Maffiuletti, N. A., Aagaard, P., Blazevich, A. J., Folland, J., Tillin, N., \& Duchateau, J. (2016). Rate of Force Development: Physiological and Methodological Considerations. European Journal of Applied Physiology, 116, 1091-1116. https://doi.org/10.1007/s00421-016-3346-6

McLester, J. R., Bishop, E., \& Guiliams, M. E. (2000). Comparison of 1 Day and 3 Days Per Week of Equal-Volume Resistance Training in Experienced Subjects. Medicine \& Science in Sports \& Exercise, 14, 273-281. https://doi.org/10.1519/00124278-200008000-00006

Merton, P. (1954). Voluntary Strength and Fatigue. The Journal of Physiology, 123, 553-564. https://doi.org/10.1113/jphysiol.1954.sp005070

Patten, C., Kamen, G., \& Rowland, D. M. (2001). Adaptations in Maximal Motor Unit Discharge Rate to Strength Training in Young and Older Adults. Muscle and Nerve, 24, 542-550. https://doi.org/10.1002/mus.1038

Place, N., Casartelli, N., Glatthorn, J. F., \& Maffiuletti, N. A. (2010). Comparison of Quadriceps Inactivation between Nerve and Muscle Stimulation. Muscle and Nerve, 42, 894-900. https://doi.org/10.1002/mus.21776

Rabita, G., Pérot, C., \& Lensel-Corbeil, G. (2000). Differential Effect of Knee Extension Isometric Training on the Different Muscles of the Quadriceps Femoris in Humans. European Journal of Applied Physiology, 83, 531-538.

https://doi.org/10.1007/s004210000325

Rhea, M. R., Alvar, B. A., Burkett, L. N., \& Ball, S. D. (2003). A Meta-Analysis to Determine the Dose Response for Strength Development. Medicine \& Science in Sports \& Exercise, 35, 456-464. https://doi.org/10.1249/01.MSS.0000053727.63505.D4

Rich, C., \& Cafarelli, E. (2000). Submaximal Motor Unit Firing Rates after 8 wk of Isometric Resistance Training. Medicine \& Science in Sports \& Exercise, 32, 190-196. https://doi.org/10.1097/00005768-200001000-00028

Rutherford, O. M., \& Jones, D. A. (1986). The Role of Learning and Coordination in Strength Training. European Journal of Applied Physiology, 55, 100-105. https://doi.org/10.1007/BF00422902

Scaglioni, G., Ferri, A., Minetti, A., Martin, A., Van Hoecke, J., Capodaglio, P., Sartorio, A., \& Narici, M. (2002). Plantar Flexor Activation Capacity and H Reflex in Older Adults: Adaptations to Strength Training. Journal of Applied Physiology, 92, 2292-2302. https://doi.org/10.1152/japplphysiol.00367.2001

Schoenfeld, B. J., Ogborn, D., \& Krieger, J. W. (2016). Effects of Resistance Training Frequency on Measures of Muscle Hypertrophy: A Systematic Review and Meta-Analysis. 
Sports Medicine, 46, 1689-1697. https://doi.org/10.1007/s40279-016-0543-8

Schoenfeld, B. J., Ratamess, N. A., Peterson, M. D., Contreras, B., \& Tiryaki-Sonmez, G. (2015). Influence of Resistance Training Frequency on Muscular Adaptations in Well-Trained Men. The Journal of Strength \& Conditioning Research, 29, 1821-1829. https://doi.org/10.1519/JSC.0000000000000970

Shield, A., \& Zhou, S. (2004). Assessing Voluntary Muscle Activation with the Twitch Interpolation Technique. Sports Medicine, 34, 253-263. https://doi.org/10.2165/00007256-200434040-00005

Shima, N., Ishida, K., Katayama, K., Morotome, Y., Sato, Y., \& Miyamura, M. (2002). Cross Education of Muscular Strength during Unilateral Resistance Training and Detraining. European Journal of Applied Physiology, 86, 287-294.

https://doi.org/10.1007/s00421-001-0559-z

Van Cutsem, M., Duchateau, J., \& Hainaut, K. (1998). Changes in Single Motor Unit Behaviour Contribute to the Increase in Contraction Speed after Dynamic Training in Humans. The Journal of Physiology, 513, 295-305.

https://doi.org/10.1111/j.1469-7793.1998.295by.x

Vangsgaard, S., Taylor, J. L., Hansen, E. A., \& Madeleine, P. (2014). Changes in H Reflex and Neuromechanical Properties of the Trapezius Muscle after 5 Weeks of Eccentric Training: A Randomized Controlled Trial. Journal of Applied Physiology, 116, 1623-1631. https://doi.org/10.1152/japplphysiol.00164.2014

Walker, S., \& Häkkinen, K. (2014). Similar Increases in Strength After Short-Term Resistance Training Due to Different Neuromuscluar Adaptations in Young an Older Men. Journal of Strength and Conditioning Research, 28, 3041-3048.

https://doi.org/10.1519/JSC.0000000000000381 